\title{
Efficient Nonadiabatic Planar Waveguide Tapers
}

\author{
Bert Luyssaert, Student Member, IEEE, Peter Bienstman, Member, IEEE, \\ Peter Vandersteegen, Student Member, IEEE, Pieter Dumon, Student Member, IEEE, \\ and Roel Baets, Senior Member, IEEE, Member, OSA
}

\begin{abstract}
We report taper designs with high transmission efficiencies and with lengths shorter than those needed for adiabatic operation. The tapering occurs between rectangular optical waveguides with the same vertical silicon-on-insulator layer structure, but with different horizontal widths, namely 0.5 and 2.0 $\mu \mathrm{m}$, and for taper lengths between 0.5 and $3.0 \mu \mathrm{m}$. After a comparison between two different optimization methods in a two-dimensional calculation scheme, one of these is repeated using three-dimensional calculations. The results show that, also in the length region where conventional linear and parabolic tapers are not yet adiabatic, tapers with a high efficiency can be designed by applying complex taper structures with more degrees of freedom.
\end{abstract}

Index Terms-Compact taper, nonadiabatic taper, optical coupler, optimization.

\section{INTRODUCTION}

$\mathbf{E}$ FFICIENT coupling between two optical waveguides has already been studied thoroughly in the past. After first proposing a linear taper [1], it was soon realized that mode conversion tends to become higher in the wider part of the taper, and applying a slower tapering there leads to tapers with parabolic shapes [2]. In [3], it was proven that when the taper length is long enough, the parabolic shape leads to the highest coupling efficiency, and a lower limit for this adiabatic operation was also given. However, this length requirement for adiabatic operation leads to very long taper structures.

Tapers are traditionally put at the edge of a photonic integrated circuit (PIC), as an interface to the outside world. Examples here are the coupling of a planar waveguide, like a laser cavity in III-V semiconductor material, to a silica optical fiber, or tapers towards the facets to ease the integration of an active PIC in III-V semiconductor material with a passive PIC in silica-on-Silicon, to create a hybrid device. In these cases, the taper, also called the spot-size convertor (SSC), normally has to change not only the size of the optical mode, but also the shape of the mode, to optimize the coupling between two different waveguides. As the number of interfaces between a PIC and the outside world is normally limited to a few, the

Manuscript received March 2, 2005; revised April 16, 2005. This work was supported in the context of the Belgian Interuniversitary Attraction Pole PHOTON network. The work of B. Luyssaert and P. Dumon was supported by the Flemish Institute for the Industrial Advancement of Scientific and Technological Research (IWT) under a specialization grant. The work of P. Bienstman was supported by the Flemish Fund for Scientific Research (FWO-Vlaanderen) through a postdoctoral fellowship.

The authors are with the Photonics Research Group, Department of Information Technology (INTEC), Ghent University-IMEC, B-9000 Gent, Belgium (e-mail: Bert.Luyssaert@intec.ugent.be; Peter.bienstman@intec. ugent.be; Peter.vandersteegen@intec.ugent.be; Pieter.dumon@intec.ugent.be; Roel.baets@intec.ugent.be).

Digital Object Identifier 10.1109/JLT.2005.850795 length of these tapers and the surface area they consume on an optical chip has never been problematic.

However, as PICs start to incorporate multiple optical components, many tapers appear within an optical chip. This occurs, for example, if photonic wires are applied where monomodality is important but broader waveguides for connections are needed to be very low loss, like in delay lines, or at the interface between photonic crystal waveguides and conventional photonic waveguides.

In an arrayed-waveguide grating (AWG), the interface between the free-space star coupler and the array waveguides can be optimized for low loss by tapering the array waveguides from touching each other while being multimodal to being decoupled and monomodal. This operation adds a large number of tapers to a PIC.

Waveguide gratings that couple light perpendicularly from a waveguide into or out of an optical fiber [5] allow the placement of optical fibers everywhere within a PIC and also make it possible to connect two-dimensional (2-D) fiber arrays directly to an optical chip. In both cases, the tapering between a broad and a narrow planar waveguide still needs to be taken care of.

As the number of tapers within a PIC grows, their footprint should become as small as possible to reduce material, processing, and packaging costs. Therefore, innovative and short taper designs are needed.

Mathematical optimization techniques have been applied before to improve the coupling between optical waveguides. In [6], the coupling between a fiber and a silica ridge waveguide is improved by $2.6 \mathrm{~dB}$ using a genetic optimization. A more mathematical approach to optimize the shape of a coupler can be found in [8], where the problem is presented as a nonlinear inverse problem. Also building on inverse problem theory is the optimization of a 3-dB Y-splitter in [9].

We will use a method similar to that of [6], but in a highcontrast index material, where other mechanisms play a role and other simulation tools must be applied.

In Section II, two methods are discussed to construct tapers and optimize them using a 2-D calculation scheme: one method based on segmented waveguide sections and one based on splines. These two methods are then compared with each other and one is selected for application in Section III, where the same optimization is then redone using an approximate threedimensional (3-D) scheme, and the results checked using a rigorous electromagnetic calculation. In Section IV, a short discussion and conclusion follows.

First structures based on the segmented waveguide approach have already been realized in silicon-on-insulator (SOI) and 


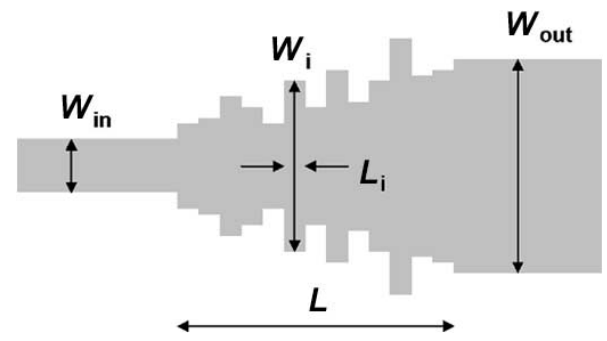

Fig. 1. Schematic presentation of the taper built out of randomly segmented waveguide sections.

have led to an improved efficiency when compared with a linear taper [10]. These structures have been designed using the same method as described in Section II, but with different widths for the input and output waveguide and with the lengths of the various waveguide segments not fixed, but also parameters to be optimized.

\section{2-D CALCULATiONS}

The effective index transformation converts a 3-D optical problem into an approximately equivalent 2-D problem. An SOI layer structure $(0.22-\mu \mathrm{m} \mathrm{Si} / 1.0-\mu \mathrm{m}$ silica/Si-substrate) results in an effective core index of 2.83 for the transverse electric (TE) mode (dominant electric field component parallel to the layer structure) at a wavelength of $1.55 \mu \mathrm{m}$.

After this transformation, 2-D structures are calculated using CAvity Modelling FRamework (CAMFR) [11], an eigenmode expansion method.

\section{A. Randomly Segmented Waveguide Approach}

Input and output waveguides are separated by a predefined distance $L$, the total length of the taper (Fig. 1), and a number $N$ of short waveguide segments all with the same length $L_{i}=$ $L / N$, along the propagation axis $z$, but with different widths $W_{i}$, along the perpendicular direction $x$, are placed in between, symmetrically around the propagation direction. The widths are bound by a lower and upper limit and are integer multiples of a small width step $\Delta L$, mostly $10 \mathrm{~nm}$. The lower limit and the step of the allowed widths are chosen to reflect the resolution of the fabrication process, while the upper width limit allows us to work with a fixed simulation area, which speeds up the numerical computation.

By changing the widths $W_{i}$ of all the sections, the ground mode intensity transmission between the two straight waveguides also changes. To find out which width values $\left[W_{1}, W_{2}, \ldots, W_{N}\right]$ lead to a good transmission, an evolutionary or genetic optimization is applied. To evaluate the transmission of a particular structure, its energy ground-mode transmission is calculated using a bidirectional fully vectorial eigenmode expansion with an absorbing boundary condition [11]. The exact workings of the method are not repeated here, but it suffices to mention that a scattering matrix is computed connecting the forward- and backward-propagating waveguide modes in the input and output waveguide. One element of this matrix is the amplitude transmission between the forward ground mode in the input and the output waveguide, and is a complex number
$T(0,0)$. The square of the modulus of this number $|T(0,0)|^{2}$ is a real number and gives the energy ground-mode transmission of the taper. This is a direct measure of the taper's efficiency and is used as its fitness in the optimization.

The optimization scheme is similar to the one explained in [6] and [7], except for the crossover operator and that a waveguide section cannot disappear during our optimization, to keep the taper length $L$ fixed. As the starting point for the optimization, 250 random structures, now called individuals are computed and their respective transmissions, or fitnesses, calculated. Then, the 100 fittest initial individuals form what is called generation 0 . Of this generation, two individuals, now called parents, are chosen using Roulette Wheel selection, where a higher intensity transmission $T$ gives a higher chance of being selected, namely $T_{j} / \sum_{i} T_{i}$. The properties of the selected individuals are mixed into two new structures, their offspring. The mixing occurs using a uniform crossover, meaning that for every section it is randomly determined whether offspring individual 1 inherits the width from parent 1 or parent 2, with offspring individual 2 always inheriting the other width. After crossover, a mutation slightly changes each offspring individual by applying a random change to every $W_{i}$. The new value of $W_{i}$ is obtained in a three-step process. It starts with a normally distributed random number with $\mu=W_{i}$ and $\sigma=0.75 \times \Delta L$, which is then rounded off to the nearest multiple of $\Delta L$, and lastly, checked whether it lies between the upper and lower limits for $W_{i}$, otherwise the nearest limit is taken. This procedure guarantees approximate chances of $50 \%$, $46 \%$, and $4 \%$ for respective width changes of $0 \cdot \Delta L, \pm 1 \cdot \Delta L$, and $\pm 2 \cdot \Delta L$ after mutation. The offspring gets calculated and added to generation 0 , of which the two individuals with the worst transmission efficiency are removed, and the remaining make up generation 1 . From here on, the same process iterates until a certain stop condition, in this case until the difference in fitness between the best and the worst (one hundredth) individual within a generation converges to less than 0.005 . This typically takes less than 1000 generations, with some exceptions (many sections and a short total length) requiring up to 2500 generations.

We studied the tapering between an input waveguide width of $0.5 \mu \mathrm{m}$ and an output waveguide width of $2.0 \mu \mathrm{m}$ for lengths of the taper section between 0.5 and $3.0 \mu \mathrm{m}$. For these widths, a linear taper is already adiabatic when $10 \mu \mathrm{m}$ long, so the footprint of a taper is already modest and the achievable gain negligible. However, this case should be seen as an example that can be ported to other coupling problems, where a bigger gain can be achieved.

For every taper length, the number of taper sections is varied from 2 to 14 in steps of two. Fig. 2 shows some of the results, with every point being the end result of a separate genetic optimization. While a smooth linear and parabolic taper of length $1.5 \mu \mathrm{m}$, have an efficiency of $84 \%$ and $80 \%$, respectively, the structure with 14 sections transmits $99 \%$ of the incoming power for an equal length.

As the degree of freedom of a structure increases, in this case meaning more sections, the efficiency also increases. The spectrum of a structure composed of 14 sections with a total length of $1.5 \mu \mathrm{m}$ is shown in Fig. 3. Fig. 4 shows the real 


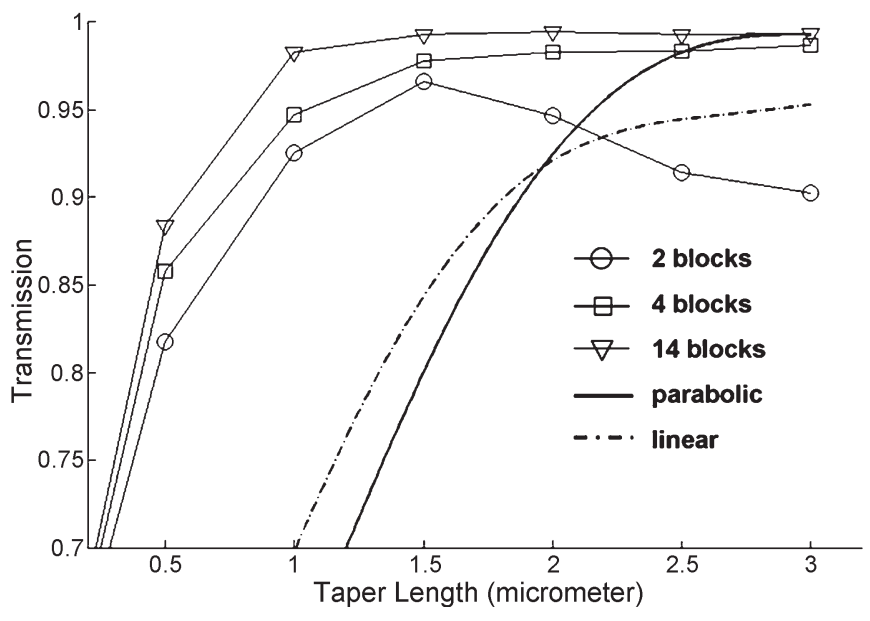

Fig. 2. Optimized efficiencies of a segmented taper as a function of length for various numbers of sections. For comparison, smooth linear and parabolic tapers are added. Width of the input and output waveguides are 0.5 and $2.0 \mu \mathrm{m}$, respectively.

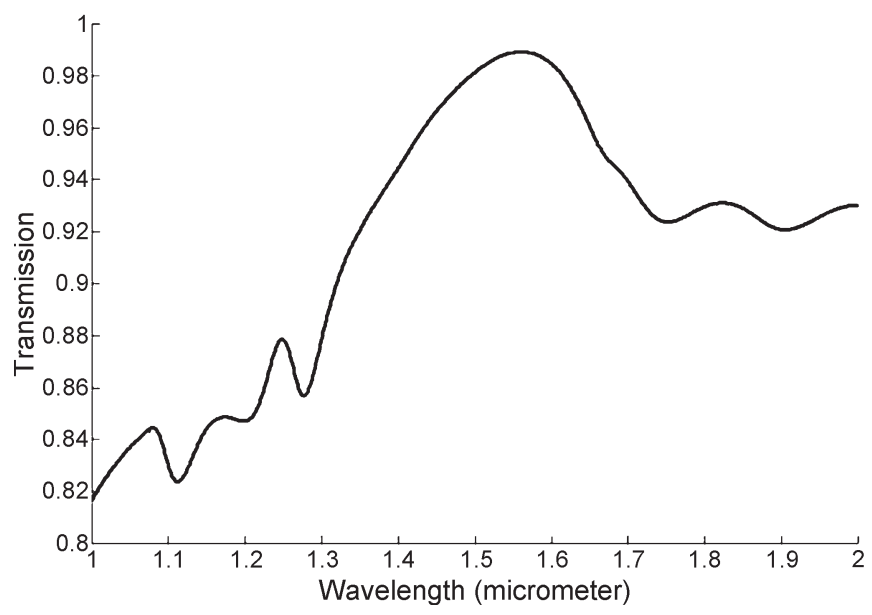

Fig. 3. Spectrum of the taper consisting of 14 blocks and with a total length of $1.5 \mu \mathrm{m}$.

part of the transverse magnetic field when the same structure mentioned previously is excited with the TE ground mode at the left. Fig. 3 reveals that the spectrum remains above $98 \%$ for wavelengths in the range 1.5-1.6 $\mu \mathrm{m}$, proving that the sharp width variations in the structure do not necessarily lead to strong resonances.

\section{B. Spline-Based Staircase Approach}

Instead of randomly placing independent waveguide segments between input and output, a taper is constructed by randomly placing a number of points within a predefined box, the length of which is the same as the taper length, and the width is defined by the minimum and maximum waveguide width allowed within a taper (Fig. 5).

The points are connected using cubic splines, which are piecewise third-order polynomials that satisfy the condition for a continuous zeroth and first derivative in each point. For the inner points, these conditions completely define each spline. At the outer points, however, an extra condition is set by forcing the second derivative to zero, which has the advantage that

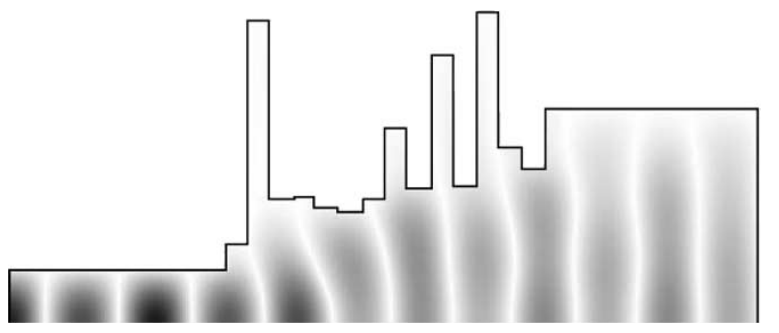

Fig. 4. Transversal magnetic field (real part) of a segmented taper excited with the ground mode at the left. The taper has a length of $1.5 \mu \mathrm{m}$, connects waveguides with widths of 0.5 and $2.0 \mu \mathrm{m}$, and consists of 14 sections.
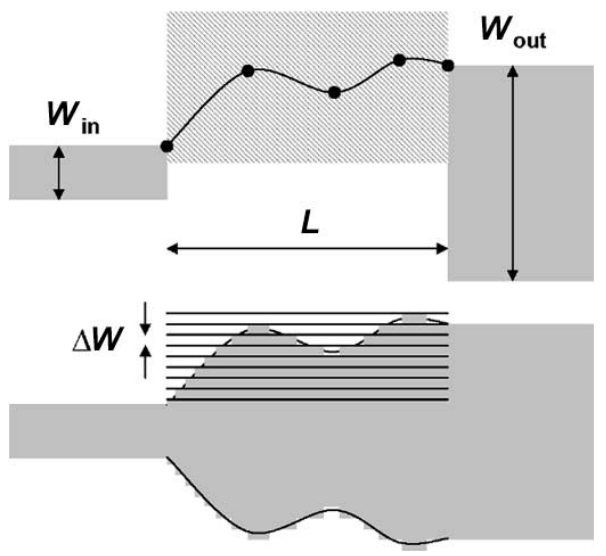

Fig. 5. Schematic presentation of the spline polynomial used to construct a taper and the staircase approximation applied before calculation.

splines with zero inner points coincide with linear tapers. The choice for a particular boundary condition does not exclude certain structures, as an extra spline point very close to a boundary point makes the condition in this point unimportant. The composed curve satisfying all these conditions is called a natural cubic spline. Again, the structures are symmetrical around the propagation ( $z$-)axis. To calculate the structure with eigenmode expansion, it needs to be divided into sections that are invariant in the propagation direction. A discretization of the continuous splines based on a fixed distance $\Delta W$, typically $12.5 \mathrm{~nm}$, in the transversal direction was chosen (Fig. 5). In what follows, the two fixed points, one at the input and one at the output, are not counted when the number of points is given.

The genetic optimization for the semicontinuous approach follows the same scheme as in the previous section, except for the crossover, where now a one-point crossover is applied to generate two new individuals. An integer number $m$ is randomly chosen between zero and the total number of points $t$. Offspring individual 1 inherits the first $m$ points of individual 1 and the last $t-m$ points of individual 2, with offspring individual 2 always inheriting the other points. Each point gets mutated using the same Gaussian distribution as before, but with $\sigma=7.5 \mathrm{~nm}$ and not discretized. Afterwards, all points of a new individual are sorted with ascending $z$-coordinates, to guarantee that the spline curve is a function.

Input and output waveguides and calculated taper lengths are similar to those in the previous section. For every taper length, the number of points is varied between zero and seven. 


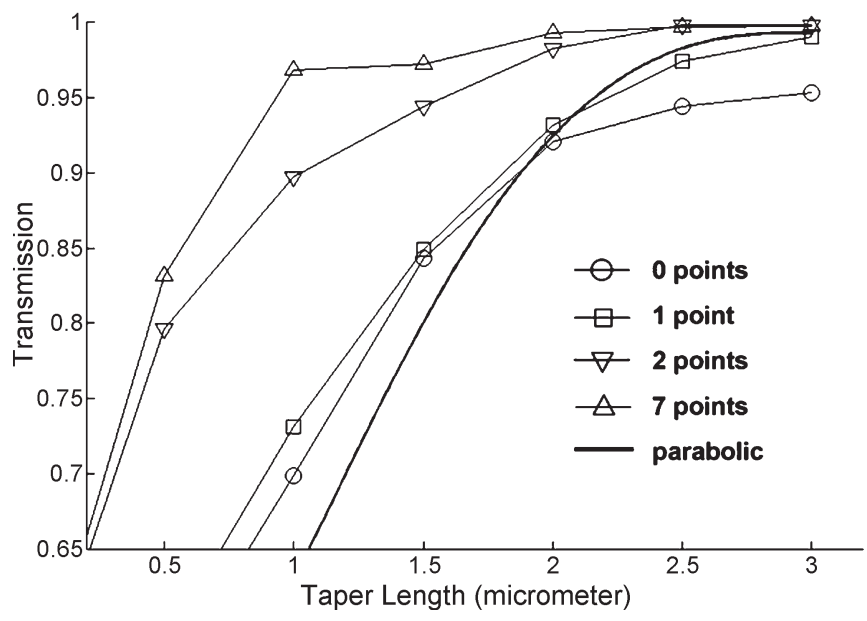

Fig. 6. Optimized efficiencies of spline tapers as a function of the taper length for various numbers of base points.

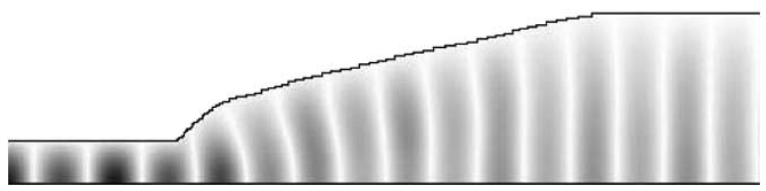

Fig. 7. Field plot corresponding to a spline taper with seven points and a length of $2.5 \mu \mathrm{m}$.

Every point in Fig. 6 is the end result of a genetic optimization. The curve labeled 0 points corresponds to a linear taper; due to the boundary conditions for natural cubic splines, the curve based on one point can never exactly coincide with a parabolic taper. Again, it can be noted that a higher degree of freedom leads to increasing efficiency.

Although a genetic algorithm does not guarantee the finding of the absolute extremum of the function under evaluation, we have two reasons to assume our optimized structures approximate this best structure very closely.

First, the shape of structures with relatively long lengths are approximately parabolic (see Fig. 7 as an example). This is to be expected of a structure whose length approaches the condition for adiabatic operation. In this figure, only half of the structure is shown because of symmetry reasons. This symmetry is also exploited in the electromagnetic calculations by placing an electric wall [11] at the position of the mirror plane.

And second, there is convergence within the results. For a fixed taper length but an increasing number of points, structures do not drastically change shape (Fig. 8). As different structures are the results of totally independent optimizations, this leads us to the conclusion that our structures converge to an optimal form that could be described by the spline approach using a large number of points.

\section{Comparison and Conclusion of 2-D Calculations}

Both 2-D approaches yield results that outperform smooth linear and parabolic tapers of equal lengths. Both cases also indicate that adding degrees of freedom to a structure leads to increased efficiency after optimization. Due to the differences between the two, rectangular blocks versus polynomial func-

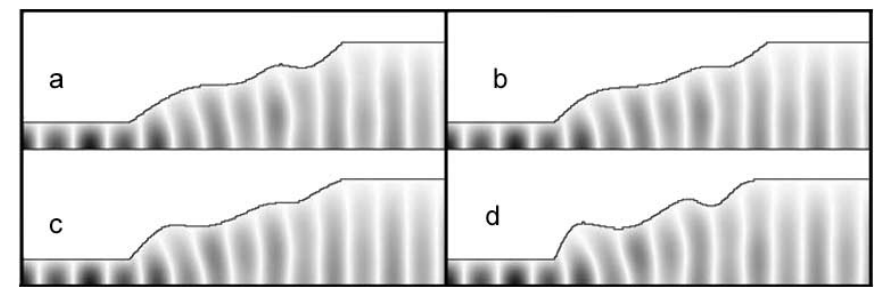

Fig. 8. Field plots of spline tapers with a total length of $2.0 \mu \mathrm{m}$ and (a) four (b) five, (c) six, and (d) seven points to illustrate the convergence of results.

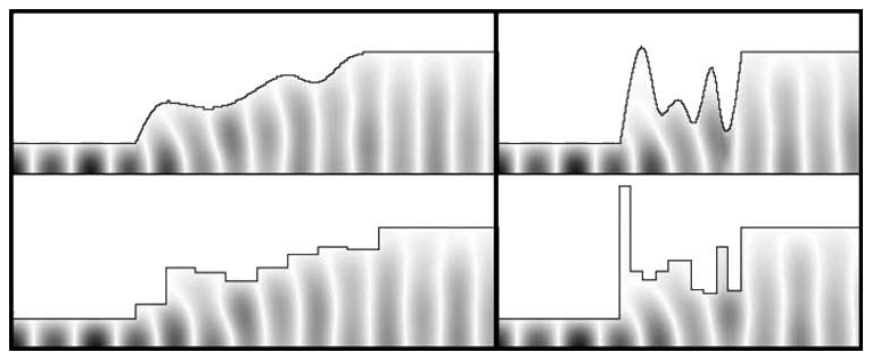

Fig. 9. Left: Spline taper with seven points and block taper with eight sections, taper length of $2.0 \mu \mathrm{m}$. Right: Spline taper with seven points and block taper with ten sections, taper length of $1.0 \mu \mathrm{m}$.

tions, a certain structure cannot exactly be described by both. However, when comparing various final structures of the two cases (Fig. 9), some appear to be similar, not only in the form of the taper but also in the resulting effect on the field. Note that the length of a section is fixed, and only the width can be altered during the course of the optimization.

In this figure, it is also clear that the shorter a certain design, the more irregular its form becomes. For longer lengths, the tapers closely resemble parabolic tapers, but when becoming shorter, bumps seem to appear in the structures. Up until now, we have not been able to mathematically describe the function of these bumps, although they seem to favor the power transfer between guided and radiation modes.

A comparison of Figs. 2 and 6 shows that the block approach yields slightly better results for a certain number of variables. As the two coordinates of a spline point can be altered, while the length of a block section is fixed and only the width is changed, one has to compare a number of spline points with the double number of block sections.

Nevertheless, we will use the spline approach in the next section for reasons of manufacturability. Fig. 10 shows both a design as it appears on a lithography mask and the resulting structure appearing in the lithographic resist layer after illumination in a deep-UV stepper at $248 \mathrm{~nm}$. The resolution of this imaging system is in the range of half the illumination wavelength and is responsible for the rounding off of the intended square structures, with some smaller artifacts almost completely disappeared. Taper forms based on splines are inherently less abrupt and should therefore be easier to manufacture.

\section{3-D CALCULATIONS}

\section{A. Unidirectional Eigenmode Expansion}

Because bidirectional 3-D eigenmode expansion calculations are still too time consuming to be useful in combination with 


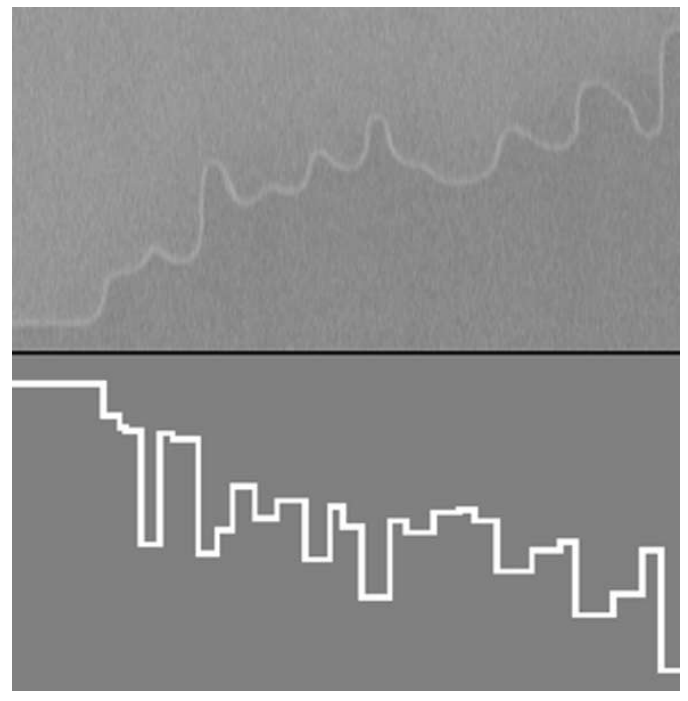

Fig. 10. Top: Scanning electron micrograph of a structure in resist. Bottom: Original mask design. Size: $6 \times 6 \mu \mathrm{m}$.

a structural optimization, a unidirectional approximation, as explained later, is applied.

The optimization scheme is exactly the same as the one explained in Section II-B, except for the calculation of a structure. An advantage of starting from a continuous taper form and of the staircase approximation based on a fixed $x$ grid is that a certain waveguide cross section can only be followed by the cross section that is $\Delta W$ narrower or wider. This drastically reduces the number of possible transitions between cross sections that occur during the course of the optimization. Preceding the optimization, a number of calculations are executed once and stored in memory afterwards. Of every possibly occurring waveguide cross section $A$, the first $N$ modes are determined and their propagation constants $\beta_{A}^{i}$ stored. Then $T_{A, B}$, the (complex) amplitude transmission matrix of the current cross section $A$ to the next cross section $B$, is calculated; element $(i, j)$ of this $N \times N$ matrix is the transmission from mode $i$ in cross section $A$ to mode $j$ in cross section $B$. Note that the matrix corresponding to the opposite situation, transmission from $B$ to $A$, can be easily deduced from the previous situation with the formula $T_{B, A}=T_{A, B}^{\mathrm{T}}$. This information suffices for the calculation of the transmission of a random structure based on a continuous function, under the assumption of negligible reflection. For every waveguide section, e.g., $C$, with a length $L$, a diagonal $N \times N$ propagation matrix $P^{C, L}$ is derived by placing $\exp \left(-j \times \beta_{C}^{i} \times L\right)$ as the $i$ th diagonal element. Suppose that a certain structure is approximated by cross section $A$, followed by $B$ with a length $L_{1}, C$ with a length $L_{2}$, again $B$ with $L_{3}$, and ends in $C$, then the total transmission matrix can be written as

$$
T=T_{A, B} \cdot P^{B, L_{1}} \cdot T_{B, C} \cdot P^{C, L_{2}} \cdot T_{C, B} \cdot P^{B, L_{3}} \cdot T_{B, C}
$$

and the ground-mode intensity transmission is equal to $|T(0,0)|^{2}$. The advantage of this scheme is the recyclability of most of the data in the form of the propagation constants and the transmission matrices. The disadvantage is that reflection is completely neglected, which has little effect on smooth, long

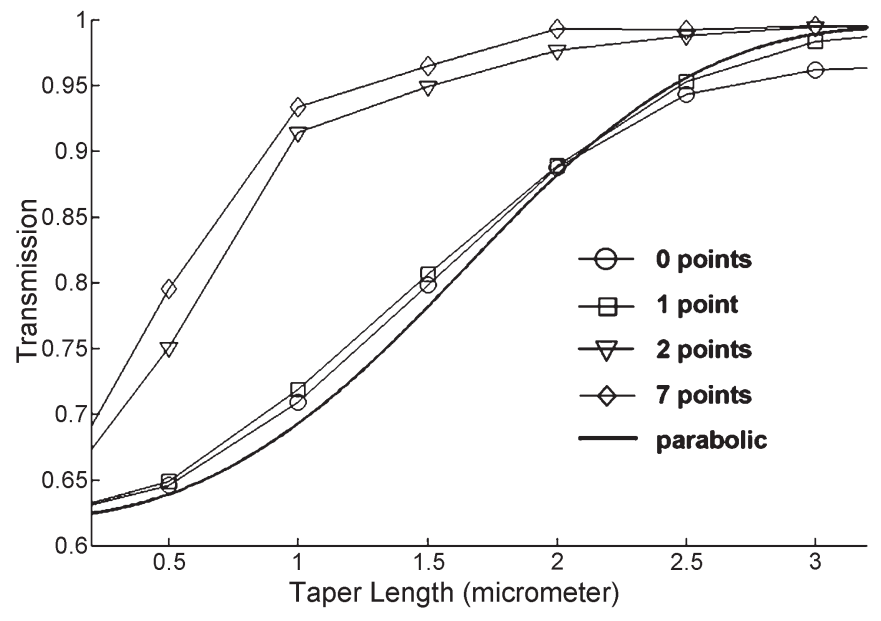

Fig. 11. Optimized efficiencies of spline tapers as a function of the taper length for various numbers of base points calculated with a 3-D unidirectional method.

tapers but increases significantly with decreasing taper length. The quantitative effect of this approximation is studied in the next section.

The resulting efficiencies as a function of taper length and for various numbers of spline points are shown in Fig. 11. What is striking in this figure is the big difference in transmission between structures based on one point and those based on two points, while adding even more points only seems to have a marginal effect as opposed to the 2-D scheme, Fig. 6, where there is also a big difference between one and two points, but where adding extra points can still bring significant improvement. This could mean that the bumps do not have a similar effect in a 3-D scheme, maybe because the radiation modes are not recaptured as well as in 2-D, where the out-of-plane radiation is not taken into account.

\section{B. Bidirectional Eigenmode Expansion}

As explained before, the optimized 3-D structures of Section III-A do not take reflection into account. Because this approximation can be rather crude for short structures, it is necessary to check the results obtained with the unidirectional method afterwards with a correct model. The model applied here is the 3-D version of CAMFR [11], essentially a fully vectorial 3-D eigenmode expansion. A complete calculation of a structure consisting of around 20 different waveguide cross sections takes a few hours of calculation time, making it impossible to use this model in a structural optimization as described above.

All structures of the previous section with lengths between 0.5 and $3.0 \mu \mathrm{m}$ and based on one to seven points are recalculated, together with the accompanying (discretized) linear and parabolic tapers (Fig. 12). For each length, the most efficient structure is shown together with the efficiency calculated with the unidirectional method. One field plot is shown as an illustration in Fig. 13. In all cases, the correct efficiency is lower than the unidirectionally calculated efficiency, with the difference being higher for shorter lengths, which points to an increasing reflection in these structures. 


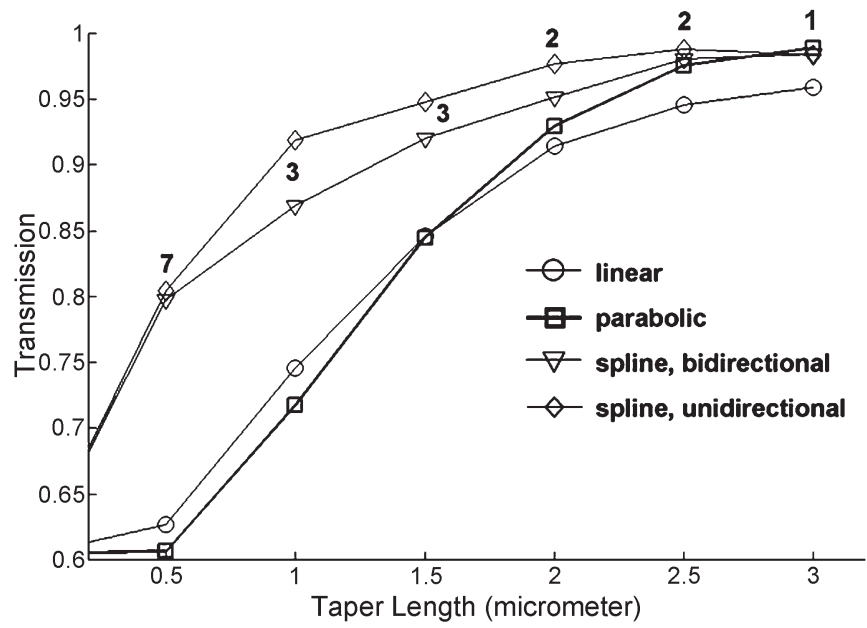

Fig. 12. Comparison between taper efficiencies calculated using 3-D unidirectional and bidirectional eigenmode expansion. The number of points to construct the taper is indicated.

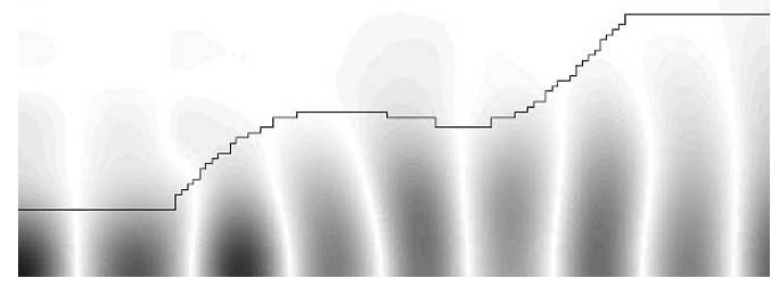

Fig. 13. Field plot (transversal magnetic field at the middle of the silicon layer) of the taper with a length of $1.5 \mu \mathrm{m}$ and based on three points.

For taper lengths too short for adiabatic operation, the optimized structures always perform better, up to $20 \%$, than parabolic and linear tapers of that length.

While adding more degrees of freedom in all previous sections increased the efficiency, this seems not to be the case here. The structure leading to the highest efficiency is almost always based on two or three points. This means that the gain achieved by refining in the unidirectional optimization is below the error induced by the approximation of that method.

\section{CONCLUSION}

We have studied the coupling between planar waveguides with different widths but the same vertical layer structure, namely silicon-on-insulator (SOI). The specific problem under study was the coupling between SOI waveguides with widths of 0.5 and $2.0 \mu \mathrm{m}$.

Two different algorithms were proposed to construct taper forms other than linear and parabolic and compared to each other using two-dimensional (2-D) calculations. Although the block-based algorithm theoretically yields better results than the spline-based approach, practical considerations have made us choose the spline-based algorithm for an implementation in a three-dimensional (3-D) calculation. To be time-efficient, a unidirectional 3-D model was applied for the optimization and afterwards checked using a full bidirectional eigenmode expansion.
For taper lengths too short for adiabatic operation, where parabolic tapers do not yet lead to a good coupling efficiency, the optimized taper forms always perform better than conventional parabolic tapers. Even for a taper length of only $1 \mu \mathrm{m}$, a transmission of $85 \%$ is reached. To achieve even better operation, we believe that an optimization using a bidirectional 3-D calculation should be applied.

\section{REFERENCES}

[1] D. Marcuse, "Radiation losses of tapered dielectric slab waveguides," Bell Syst. Tech. J., vol. 49, no. 2, pp. 273-290, 1970.

[2] C. K. Burns, A. F. Milton, and A. B. Lee, "Optical-waveguide parabolic coupling horns," Appl. Phys. Lett., vol. 30, no. 1, pp. 28-30, 1977.

[3] A. F. Milton and C. K. Burns, "Mode coupling in optical waveguide horns," IEEE J. Quantum Electron., vol. 13, no. 10, pp. 828-835, Oct. 1977.

[4] K. Kato and Y. Tohmori, "PLC hybrid integration technology and its application to photonic components," IEEE J. Sel. Top. Quantum Electron., vol. 6, no. 1, pp. 4-13, Jan.-Feb. 2000.

[5] D. Taillaert, P. Bienstman, and R. Baets, "Compact efficient broadband grating coupler for silicon-on-insulator waveguides," Opt. Lett., vol. 29, no. 23, pp. 2749-2751, 2004.

[6] M. M. Spühler, B. J. Offrein, G. L. Bona, R. Germann, I. Masserek, and D. Erni, "A very short planar silica spot-size converter using a nonperiodic segmented waveguide," J. Lightw. Technol., vol. 16, no. 9, pp. 1680-1685, Sep. 1998.

[7] M. M. Spühler, D. Erni, and J. Fröhlich, "An evolutionary optimization procedure applied to the synthesis of integrated spot-size converters," Opt. Quantum Electron., vol. 30, no. 5-6, pp. 305-321, 1998.

[8] T. Felici and H. W. Engl, "On shape optimization of optical waveguides using inverse problem techniques," Inverse Probl., vol. 17, no. 4, pp. 1141-1162, 2001.

[9] T. Yabu, M. Geshiro, and S. Sawa, "New design method for low-loss Y-branch waveguides," J. Lightw. Technol., vol. 19, no. 9, pp. 1376-1384, Sep. 2001.

[10] B. Luyssaert, P. Vandersteegen, D. Taillaert, P. Dumon, W. Bogaerts, P. Bienstman, D. Van Thourhout, V. Wiaux, S. Beckx, and R. Baets, "A compact photonic horizontal spot-size converter realized in silicon-oninsulator," IEEE Photon. Technol. Lett., vol. 17, no. 1, pp. 73-75, Jan. 2005.

[11] P. Bienstman and R. Baets, "Optical modelling of photonic crystals and VCSELs using eigenmode expansion and perfectly matched layers," Opt. Quantum Electron., vol. 33, no. 4-5, pp. 327-341, 2001.

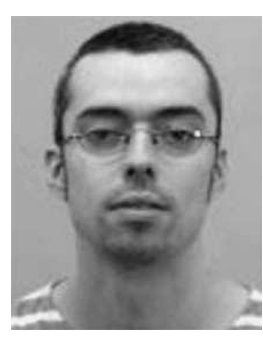

Bert Luyssaert (S'01) was born in Gent, Belgium, in 1976. He received the B.S. degree in physics and the B.S. degree in engineering (physics) from Ghent University, Gent, Belgium, in 1998 and 2000, respectively. $\mathrm{He}$ is currently working toward the $\mathrm{Ph} . \mathrm{D}$. degree in engineering at the Department of Information Technology, Ghent University.

$\mathrm{He}$ has research interests in the design, fabrication, and characterization of components for microphotonics, and in particular, coupling problems between various components.

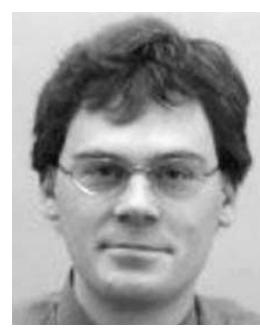

Peter Bienstman (S'97-A'01-M'03) was born in Ghent, Belgium, in 1974. He received the B.S. degree in electrical engineering and the Ph.D. degree from Ghent University, Gent, Belgium, in 1997 and 2001, respectively.

From 2001 to 2002, he was with the Joannopoulos Research Group, Massachusetts Institute of Technology, Cambridge. He is currently an Assistant Professor with the Department of Information Technology (INTEC), Ghent University. His research interests include the modeling of optical structures, notably photonic crystal structures, vertical-cavity surface-emitting lasers, and resonant-cavity light-emitting diodes. He has published several papers and has one patent. 


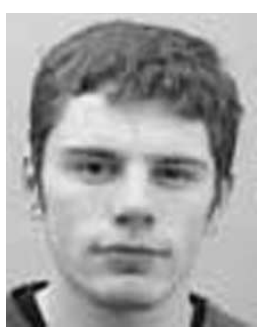

Peter Vandersteegen ( $\left.\mathrm{S}^{\prime} 03\right)$ was born in Bree, Belgium, in 1980. He received the B.S. degree in physical engineering from Ghent University, Gent, Belgium, in 2003. He is currently working toward the $\mathrm{Ph} . \mathrm{D}$. degree in engineering at the Department of Information Technology, Ghent University.

He has for his main research interest the development of simulation tools for optical structures.

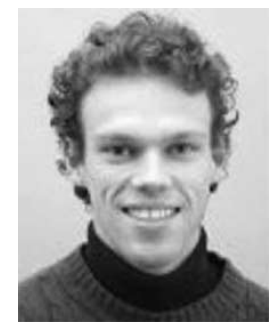

Pieter Dumon (S'02) received the B.S. degree in electrical engineering from Ghent University, Gent, Belgium, in 2002, where he is currently working toward the $\mathrm{Ph} . \mathrm{D}$. degree in electrical engineering.

$\mathrm{He}$ has research interests in the modeling, design, and fabrication of nanophotonic waveguides and structures for passive photonic integrated circuits.

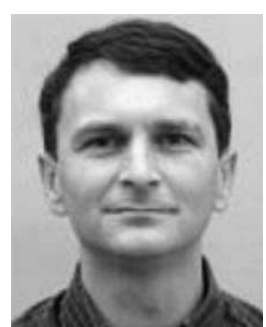

Roel Baets (M'88-SM'96) received the B.S. degree in electrical engineering from Ghent University, Gent, Belgium, in 1980, the M.Sc. degree in electrical engineering from Stanford University, Stanford, $\mathrm{CA}$, in 1981, and the Ph.D. degree from Ghent University, in 1984.

He has been with the Department of Information Technology (INTEC) of Ghent University since 1981, where he has been a Professor in the engineering faculty since 1989. From 1990 to 1994, he was also a part-time Professor at the Technical University of Delft, Delft, The Netherlands. He has mainly worked in the field of photonic components. With about 300 publications and conference papers, as well as about ten patents, he has made contributions to the design and fabrication of III-V semiconductor laser diodes, passive guided-wave devices, photonic ICs, and microoptic components. He leads the Photonics Group at Ghent University-INTEC, which is an associated laboratory of the Interuniversity Microelectronics Center (IMEC), Leuven, Belgium, working on integrated photonic devices for optical communication, optical interconnect, and optical sensing.

Dr. Baets is a Member of the Optical Society of America (OSA), The International Society for Optical Engineers (SPIE), and the Flemish Engineers Association. He has been a Member of the program committees of Optical Fiber Communication Conference (OFC), European Conference on Optical Communication (ECOC), IEEE Semiconductor Laser Conference, European SolidState Device Research Conference (ESSDERC), Conference on Lasers and Electro-Optics (CLEO)-Europe, and the European Conference on Integrated Optics. He has been chairman of the IEEE-Lasers \& Electro-Optics Society (LEOS)-Benelux Chapter from 1999 to 2001. Currently, he is a Member of the Board of Governors of IEEE-Lasers \& Electro-Optics Society (LEOS). 